\title{
An epidemiological study of clinic attendees at the first specialised clinic for individuals with intellectual disability in Sri Lanka \\ P Ginige, WHMKJ Wijesinghe, SUB Tennakoon
}

\section{Background}

Intellectual disability (ID) is diagnosed when both intellectual and adaptive functioning deficits are shown in conceptual, social and practical domains during the developmental period. A higher prevalence of ID in areas of lower socioeconomic status and developing countries is noted. No published studies of ID were found for Sri Lanka.

Aims

To describe the epidemiology and pattern of health seeking behaviour of individuals with ID attending a specialised clinic.

\section{Methods}

Data were extracted from the records of all individuals registered at the specialised clinic for mentally retarded individuals at Teaching Hospital, Peradeniya, from 1984 to 2013.

Results

One thousand three hundred and thirty five patient records were perused. The mean age at first presentation was 11.7 years. The majority (60.7\%) were males. Mild, moderate and severe ID was diagnosed in $78.9 \%, 15.6 \%$ and $5.5 \%$ respectively. The main presenting problem was poor scholastic abilities (54.8\%). Epilepsy was the main associated medical condition. Activities of daily living (ADLs) were impaired in $31.8 \%$ of subjects. A majority-received no behavioural interventions.

\section{Conclusion}

There was a 33-fold increase in help seeking behaviour, over the 29 year study period. The findings of this study highlight the epidemiology of ID in Sri Lanka, and the need for more clinics and a national policy for ID.

Key words: Intellectual Disability (ID), epidemiological data, specialised clinic for ID, health-seeking patterns

SL J Psychiatry 2016; 7(2): 8-11

\section{Introduction}

Intellectual Disability (ID) is the term used in the Diagnostic and Statistical Manual of Mental Disorders $5^{\text {th }}$ edition (DSM-5), for the condition previously known as mental retardation in DSM-IV. ID is a heterogeneous condition with multiple causative factors, both environmental and genetic (1-4). An individual with ID suffers mild or substantial limitations in functioning based on the degree of disability. It is characterized by significant limitations in intellectual functioning and co occurring of related limitations in two or more of the adaptive skills, such as attending to activities of daily living, communication, skills for social interaction and idea of safety and risk (5).

The estimated prevalence rates for ID vary between 3-6/ 1000 among adults and 3-14/1000 among children according to large population-based studies. The majority (75-90\%) has mild ID. It is reported that areas with lower socioeconomic status and developing countries have a higher prevalence of ID, particularly mild ID (1, 6-9). Gender ratios vary widely in published studies. Yet males are more likely than females to be diagnosed with both mild (male: female ratio 1.6:1) and severe (male: female ratio 1.2:1) forms of ID.

Published data on the pattern of ID is scarce in South Asia and no published data was found for Sri Lanka in Google Scholar and PubMed from 1980 to date. The Department of Census and Statistics of Sri Lanka, in a survey in 2001 estimated 1.6\% of Sri Lankan population were suffering from ID (10). Data on ID is beneficial to address its prevention, management and especially to improve the quality of life of the affected individuals and their families through policies on service provision (11). The objectives of this study were to describe the epidemio-logical profile of the patients registered at the specialised clinic for ID at Teaching Hospital Peradeniya (THP) and to investigate the trends in help seeking behaviour from the time of inception of the clinic to 2013. 


\section{Methods}

Ethical clearance was obtained from the Ethics Review Committee of the Faculty of Medicine, University of Peradeniya. The study was conducted at the specialised clinic for ID at THP which is the first and the only clinic for individuals with ID in SL. The routine clinic procedure is that following the registration of the subjects, a detailed assessment of the patient is carried out and the consultant psychiatrist makes the diagnosis of ID and categorises the condition as mild, moderate or severe according to the International Classification of Diseases - tenth version (ICD-10) (12). The information is recorded and archived confidentially.

All patients registered at the clinic from the year of inception of the clinic in 1983, to 31 ${ }^{\text {st }}$ of December 2013 were included in the study. There were no exclusion criteria. Epidemiological data for the study was collected from the clinic records and analysed using SPSS 17.1 .

\section{Results}

The total number of subjects registered at the clinic during the 29 years was 1335 . Males comprised $60.7 \%$ of the sample. The mean age at first presentation was 11.7 years (range 2 to $60 \mathrm{yrs}, \mathrm{SD}=7.24 \mathrm{yrs}$, median=10 yrs).

Mild ID was diagnosed in1053 (78.9\%), moderate ID in 208 (15.6\%) and severe ID in 74 (5.5\%) subjects of the study population according to ICD-10.

Out of the total of 1335, the majority (73.7\%) did not have any notable perinatal complications. However $14.6 \%$ received treatment at a neonatal care unit for perinatal problems.

The ability of looking after self without any support was limited in 425 individuals (31.8\%). The individuals who were independent in ADLs, presented late compared to individuals who were dependent on others for ADLs.

\begin{tabular}{|c|c|c|}
\hline Ability for ADLs & N (\%) & $\begin{array}{l}\text { Mean age in } \\
\text { years at } \\
\text { presentation } \\
\text { (SD) }\end{array}$ \\
\hline Independent & $908(68.0)$ & $12.9(7.1)$ \\
\hline Totally dependent & $207(15.5)$ & $9.2(6.3)$ \\
\hline $\begin{array}{l}\text { Needs help/ } \\
\text { supervision }\end{array}$ & $218(16.3)$ & $9.0(6.3)$ \\
\hline$P$ (ANOVA) & & $>0.000$ \\
\hline Missing data & $2 \quad(0.1)$ & \\
\hline Total & $1335(100)$ & \\
\hline
\end{tabular}

The difference between the mean ages of the two categories is significant (Table 1).

Poor scholastic abilities was the commonest presenting problem, accounting to $54.7 \%$ of the total presentations. Challenging behaviours such as aggression was the reason for presentation in $27.1 \%$. Irritability and hyperactivity were other notable presenting problems. Speech delay accounted for $2.7 \%$ of the presentations.

Epilepsy was the main medical condition associated with our study group with 323 (24.2\%) individuals presenting with co morbid epilepsy. Urinary incontinence was troublesome in 54 (4\%) individuals. The majority, 947 (70.9\%), did not have any associated medical problem.

Only 2 patients had registered at the clinic in 1984, (one year after the inception of the clinic in 1983), whereas 66 registered in 2013. Help seeking had increased significantly over time, since commencement of the service (Figure 1).

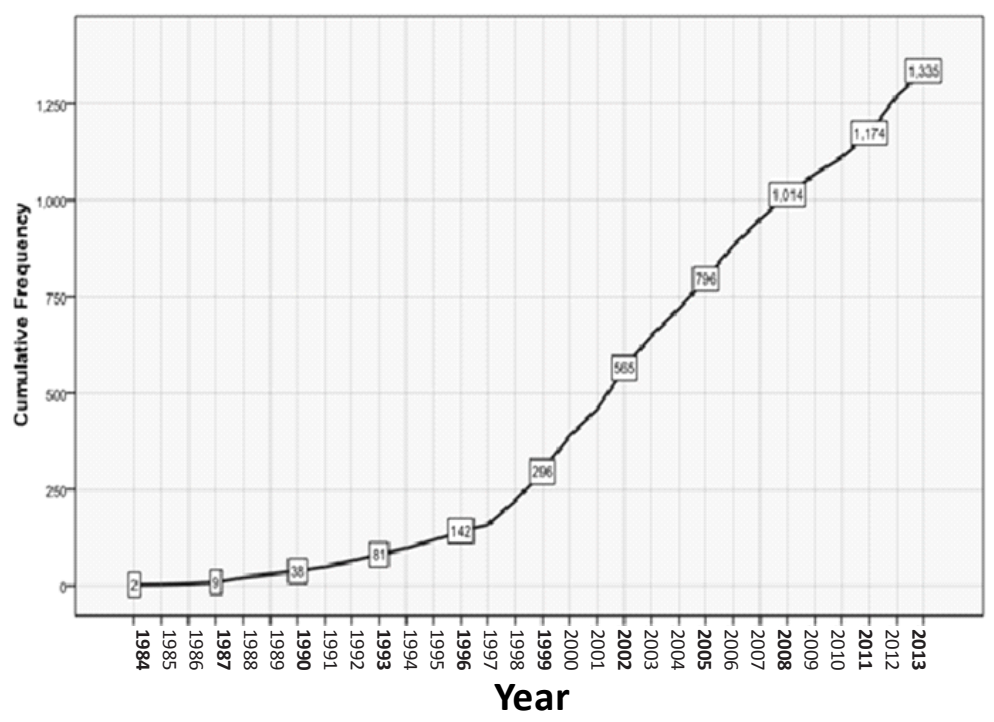

Figure 1. Help seeking pattern over 29 years. 
Patients had come to the clinic from 18 districts out of the total 24 districts in the country. Most came from Kandy, with a considerable number also coming from Kegalle (173), Nuwara Eliya (97), Matale (75), Kurunegala (35), Polonnaruwa (20), Anuradhapura (12), Ampara (7) and Badulla (6).

The majority of persons in the study had received no therapeutic interventions such as behavioural therapy, speech therapy or occupational therapy. Their families looked after most of the patients, with only 15 individuals (1.1\%) receiving residential care.

\section{Discussion}

Intellectual disability is an important public health concern in Sri Lanka, both in terms of prevalence and resultant disability. Yet, the impact of ID on the individual, family and the community is a much-neglected area. The present study was designed to highlight the epidemio-logical data of the population presenting to a specialised clinic for ID, and to describe the health-seeking trend over the years. To our knowledge this is the first such study in Sri Lanka.

The prevalence rates of mild and severe ID in the current study are similar to global data, with a majority (78.9\%) suffering from mild ID. The male to female ratio shows a male preponderance, similar to previous studies $(13,14)$. The clinical experience of the authors is that the older individuals present to services more for social benefits than for assessment or intervention.

The mean age of presentation was 11.7 years, and the commonest presenting problem was poor school performances. It is likely that this late presentation age of 11-12 years is linked to the commonest presenting problem of poor schoolwork - since this is an age at which the school curriculum becomes more complex $\left(6^{\text {th }}\right.$ and $7^{\text {th }}$ grades). Children with mild ID show major deficiencies mainly in scholastic abilities, whereas the other areas such as ADL $s$ and extended ADLs are usually age appropriate $(14,15)$. The Sri Lankan culture places great emphasis on education and the prime goal of an average parent is to give the children a good education. The mild ID children may be detected only when they struggle with academic work in grade 6 and 7.

Another important finding of the study is that the individuals who cannot perform ADLs present significantly earlier than those who can. It can be inferred that the performance on ADLs is a deciding factor for the time of presentation for help.

The concept of ID is somewhat an alien entity in the local culture. It is not uncommon to find people spending fortunes to seek a "cure” for ID or confusing it with major mental illnesses. Parents who do not recognise their child as being low in intelligence may neglect seeking intervention until the child develops behavioural problems.
Aggressive behaviour was the second common presenting problem in this study, and is obviously an important motivation for seeking help.

Late presentation poses many disadvantages to the affected child, as he or she loses the benefits of early therapeutic interventions such as training in self-help skills, and remedial teaching. Early presentation will also increase early detection and treatment of associated psychiatric co-morbidities (e.g. hyperactivity and conduct features), if present.

Help seeking behaviour has clearly increased over the last 3 decades. This could be due to raised awareness on ID. This study highlights the lack of multidisciplinary team members such as occupational therapists and speech therapists, to deliver necessary therapies at the clinic. However the clinic collaborates with local nongovernmental organizations (NGOs) that have resources and experience in caring for ID individuals and obtain their support in delivering the interventions to clinic patients.

There is no government run residential facilities for ID individuals in the country. A few scattered charity organizations and NGOs are attempting to fill the void, with various levels of success; but, as shown by the findings of this study, the brunt of caregiving falls on the family. As a result, parents often have serious concerns about the future care and wellbeing of their children with ID, after their demise.

\section{Conclusions}

The most common problem that individuals with ID present with, is difficulty in education. Aggression and associated medical problems also play a role in help seeking behaviour. About one third need some support in ADLs. Help seeking behaviour has increased 33 fold from 1983 to 2013. The authors believe that the findings of this study will be useful in creating awareness among not only general public, but also the authorities, in order to guide policy making and developing services in this much needed field.

\section{Limitations}

Detailed psychometric measurements could not be performed on 1119 patients due to there being no qualified educational psychologist at the clinic. A psychologist who was working on a volunteer basis performed detailed assessments on 216 patients. A nursing officer trained in intelligent quotient (IQ) assessment conducted basic assessments.

\section{Recommendations}

The authors recommend the establishment of clinics for individuals with intellectual disability throughout the country, and improvement of infrastructure of 
these clinics, such as employment of essential multidisciplinary team members. Such an approach would provide better access to health in even remote villages in the country. This underscores the need of national guidelines for management of ID in Sri Lanka, through collaboration between the Ministry of Health and relevant professional bodies such as the Sri Lanka College of Psychiatrists and Sri Lanka College of Paediatricians.

\section{Acknowledgements}

Drs. Helani Alahakoon, Kamal Bandara, Prarthana Liyanarachchi and Priyalini Rajmohan are greatly appreciated for the support they extended for this study.

\section{Conflicts of interest}

None declared

P Ginige, Department of Psychiatry, Faculty of Medicine, University of Peradeniya

WHMKJ Wijesinghe, National Institute of Mental Health, Angoda

SUB Tennakoon, Department of Community Medicine, Faculty of Medicine, University of Peradeniya

Corresponding author: SUB Tennakoon

Email: sampathte@yahoo.com

\section{References}

1. Drews CD, Yeargin-Allsopp M, Decoufle P, Murphy CC. Variation in the influence of selected sociodemographic risk factors for mental retardation. Am J Public Health 1995; 85 (3): 329-34.

2. Jeewanandam L. Perspectives of intellectual disability in Asia: epidemiology, policy, and services for children and adults. Curr Opin Psychiatry 2009; 22(5): 462-8.
3. Vashist M, Yadav R. Study of maternal age, family history of mental retardation, consanguinity in mental retardation. Res J of Biol 2011; 1(1): 7-10.

4. Rauch SL, Shin LM, Phelps EA. Neurocircuitry models of posttraumatic stress disorder and extinction: human neuroimaging research - past, present, and future. Biol Psychiatry 2006; 60(4): 376-82.

5. American Psychiatric Association. Diagnostic and statistical manual of mental disorders. 5th ed. Arlington, VA: American Psychiatric Publishing, 2013.

6. Roeleveld N, Zielhuis GA, Gabreels F. The prevalence of mental retardation: a critical review of recent literature. Dev Med Child Neurol 1997; 39(2): 125-32.

7. Durkin MS, Hasan ZM, Hasan KZ. Prevalence and correlates of mental retardation among children in Karachi, Pakistan. Am J Epidemiol. 1998;147(3): 281-8.

8. Durkin M. The epidemiology of developmental disabilities in low-income countries. Ment Retard Dev Disabil Res Rev. 2002; 8(3): 206-11.

9. Emerson E. Poverty and people with intellectual disabilities. Ment Retard Dev Disabil Res Rev 2007; 13(2): 107-13.

10. Department of Census and Statistics [homepage on internet]. Information on disabled persons, census of population and housing 2001, Colombo c2016; Available at: http://www.statistics.gov.lk/page.asp?page= Population \%20and\%20Housing (Accessed on 15 August, 2016).

11. Fujiura GT, Parish SL. Emerging policy challenges in intellectual disabilities. Ment Retard Dev Disabil Res Rev 2007; 13(2): 188-94.

12. World Health Organization (WHO). The ICD-10 classification of mental and behavioural disorders: clinical descriptions and diagnostic guidelines. Geneva: WHO, 1992.

13. Gissler M, Jarvelin MR, Louhiala P, Hemminki E. Boys have more health problems in childhood than girls: followup of the 1987 Finnish birth cohort. Acta Paediatrica 1999; 88 (3): 310-4.

14. Leonard H, Wen X. The epidemiology of mental retardation: challenges and opportunities in the new millennium. Ment Retard Dev Disabil Res 2002; 8(3): 117-34.

15. Goodman R, Scott S. Child and Adolescent Psychiatry. 3rd ed. Oxford: Wiley-Blackwell, 2012. 